Original

\title{
Effect of ultrasonication with EDTA or MTAD on smear layer, debris and erosion scores
}

\author{
Bahareh Dadresanfar ${ }^{1)}$, Zohreh Khalilak ${ }^{1)}$, Abbas Delvarani ${ }^{1)}$, Payman Mehrvarzfar ${ }^{1)}$, \\ Mehdi Vatanpour ${ }^{1)}$ and Mahsa Pourassadollah ${ }^{2)}$ \\ ${ }^{1)}$ Department of Endodontics, Dental Branch, Islamic Azad University, Tehran, Iran \\ ${ }^{2)}$ Private practice, Tehran, Iran
}

(Received 28 June and accepted 16 December 2010)

\begin{abstract}
The purpose of this study was to compare the effects of ultrasonication with ethylenediaminetetraacetic acid (EDTA) and a mixture of tetracycline isomer, an acid, and a detergent (MTAD) as final canal irrigants on the smear layer, debris and erosion scores. Fifty-eight extracted single-rooted human teeth were instrumented with ProTaper rotary files up to size F3. According to the final irrigation regimen, the samples were distributed into the following groups: EDTA, MTAD, EDTA ultrasonicated for 1 min, and MTAD ultrasonicated for $1 \mathrm{~min}$. The smear layer, debris and erosion scores were recorded at the apical, middle, and coronal third of each canal using a scanning electron microscope. Data were subjected to statistical evaluation using the Kruskal-Wallis and Mann-Whitney tests $(P<\mathbf{0 . 0 5})$. There were no significant differences in smear layer or debris removal between the experimental groups. EDTA caused significantly more erosion at the middle level than MTAD. Also EDTA resulted in more erosion at the coronal level than MTAD when subjected to ultrasonication. Ultrasonic activation of EDTA significantly increased its erosive effects at the middle and coronal levels. Based on the present findings, MTAD appears to cause less dentinal erosion while allowing proper removal of the smear layer and debris.
\end{abstract}

Correspondence to Dr. Zohreh Khalilak, Department of Endodontics, Dental Branch, Islamic Azad University, Number 3, 10th Neyestan Street, Pasdaran Avenue, Tehran, Postal Code: 19468, Iran

Tel: +98-21-22564571

Fax: +98-21-22564577

E-mail: dr.z.khalilak@gmail.com
(J Oral Sci 53, 31-36, 2011)

Keywords: EDTA; irrigation; MTAD; smear layer; ultrasound.

\section{Introduction}

The main objective of root canal treatment is to clean and shape the root canal system. Studies have shown that rotary instrumentation techniques produce a smear layer that covers the dentinal tubules (1). It remains controversial whether this layer should be removed or not (2). The presence of a smear layer can inhibit the flow of disinfectants and medicaments into dentinal tubules and compromise the seal of a root canal filling (3). Final canal irrigation with ethylenediaminetetraacetic acid (EDTA) and sodium hypochlorite $(\mathrm{NaOCl})$ has been recommended for removal of inorganic and organic components of the smear layer (4-6). The adverse effects of this combination on dentinal tubules and the resulting erosion of intra-radicular dentin have been reported (1,5-7).

BioPure MTAD has been introduced to dentistry as a final irrigant for smear layer removal (1). MTAD has been proved to be effective in eliminating resistant microorganisms and providing sustained antimicrobial activity $(8,9)$. Minimal erosion of intra-radicular dentin has been reported after final canal irrigation with MTAD (10).

The flushing action of the irrigating solution plays an important role during the canal cleaning and shaping process (11). Most of the dentin debris is inorganic matter and its removal relies mostly on the flushing action of the irrigant (12), and ultrasonic agitation of irrigating solutions enhances their flushing action (13-15). Based on a literature 
review by Van der Sluis et al. (15) passive ultrasonic irrigation is regarded as an adjunctive treatment for root canal cleaning, and is more effective than syringe irrigation. It has been shown that the use of ultrasonication with 17\% EDTA improves smear layer removal in the apical region of the canal (16).

The aim of the present study was to compare the in vitro effect of ultrasonication with EDTA and MTAD as the final canal irrigants on smear layer, debris and erosion scores at the coronal, middle, and apical levels of extracted human teeth.

\section{Materials and Methods}

Fifty-eight extracted mature maxillary and mandibular single-rooted non-carious human teeth with a curvature of less than $20^{\circ}$ (17) were used for this study. Teeth with previous coronal restorations or root canal treatment were excluded. A conventional access cavity was prepared for each tooth. The working length was determined by reducing $1 \mathrm{~mm}$ from the length recorded when a \#10 k-file (Dentsply, Maillefer, Balligues, Switzerland) was placed through the apical foramen. The specimens were instrumented with ProTaper rotary files (Dentsply) up to size F3 as Master Apical Rotary (MAR). An X-smart endodontic motor controller (Dentsply) was used to control the speed and torque for each file according to the specifications of the manufacturer. The canals were irrigated with $2 \mathrm{ml}$ of $2.6 \%$ $\mathrm{NaOCl}$ between every instrument change by means of a 28-gauge prorinse probe syringe (Dentsply, Tulsa, USA).

The teeth were randomly distributed in 4 groups of 12 . The remaining 10 teeth were distributed into positive and negative control groups each including 5 teeth. Teeth in the positive control group were instrumented in the same way as samples in the experimental groups, except that the irrigant used between each instrumentation and as the final rinse was distilled water. In this way the smear layer remained intact on the canal wall. The samples in the negative control group received no instrumentation and were rinsed with $1 \mathrm{ml}$ EDTA for $1 \mathrm{~min}$ and then $5 \mathrm{ml}$ of $2.6 \% \mathrm{NaOCl}$ to ensure absence of the smear layer on the canal walls.

\section{Final irrigation sequence}

The final irrigation sequences were as follows:

Group A, $1 \mathrm{ml}$ EDTA for $1 \mathrm{~min}$ followed by $5 \mathrm{ml}$ of $2.6 \%$ $\mathrm{NaOCl}$; Group B, 1 ml MTAD (Dentsply, Tulsa, USA) for 5 min, activated by a \#15K-file, then rinsed with $4 \mathrm{ml}$ MTAD; Group C, 1 ml EDTA ultrasonicated by a \#15 Ufile for $1 \mathrm{~min}$. The ultrasonic unit used was a Varios 350 (NSK Nakanishi Inc., Kanuma, Japan) and the power adjustment on the unit was set at 3 according to the manufacturer's specification; group D, $1 \mathrm{ml}$ MTAD for 5 min, ultrasonicated in the same manner as group $\mathrm{C}$ for the last $1 \mathrm{~min}$, followed by a rinse with $4 \mathrm{ml}$ MTAD. The canals were dried with a paper point (Gapadent Co., Tianjin, China) and longitudinal grooves were cut in the buccal and lingual surfaces of the roots with a diamond disc (D\&Z, Berlin, Germany), taking care not to penetrate the root canals. The roots were then split into two halves with a chisel, and each half root was placed in $2.5 \%$ glutaraldehyde for $24 \mathrm{~h}$. The fixed specimens were rinsed with phosphate buffer solution (0.1 M, pH 7.2) three times, incubated in osmium tetroxide for $2 \mathrm{~h}$, dehydrated with ascending concentrations of ethyl alcohol (50-100\%), and placed in a desiccator for 24-48 $\mathrm{h}$. The specimens were mounted on an aluminum stub and coated with gold-palladium to a thickness of $550 \AA$ A using a SCD 005 sputter coater (BalTEC, Pfäffikon, Switzerland). Specimens were then examined using a scanning electron microscope (Leo440h, Oxford, UK) at a magnification of $\times 1,000$ at the apical, middle, and coronal thirds of each canal. In a blind manner, three investigators scored the SEM photographs separately. Before the proper scoring, all of the examiners assessed the first 20 specimens together for calibration purposes. Separate evaluations were recorded for the smear layer and debris according to the following schemes (18):

Smear layer scale:

1: no smear layer, orifices of the dentinal tubules patent.

2: small amount of smear layer, some open dentinal tubules.

3: homogeneous smear layer along almost the entire canal wall, with only very few open dentinal tubules.

4: the entire root-canal wall covered with a homogeneous smear layer, with no open dentinal tubules.

5: a thick homogenous smear layer covering the entire rootcanal wall.

Debris scale:

1: clean canal wall, only very few debris particles.

2: many conglomerations.

3: many conglomerations; less than $50 \%$ of the canal wall covered.

4: more than $50 \%$ of the canal wall covered.

5: complete or nearly complete covering of the canal wall by debris.

The scoring system used for the degree of dentinal tubule erosion was as follows (10):

1: No erosion. All tubules normal in appearance and size.

2: Moderate erosion. Peritubular dentin eroded.

3: Severe erosion. Intertubular dentin destroyed, and tubules connected to each other.

Data were subjected to statistical evaluation using the Kruskal-Wallis and Mann-Whitney tests; $P$ values were computed and compared for statistical significance at the 
$P=0.05$ level.

\section{Results}

Kappa test for the first 20 samples showed high intraand inter-examiner agreement values ( 0.8 and above).

The mean ranks for the smear layer, debris and erosion in each group are presented in Figs. 1, 2 and 3, respectively.

A comparison of the smear layer covering dentinal surfaces at the apical, middle and coronal levels showed no significant differences between the experimental groups. The difference between the positive control group and the experimental groups was significant $(P=0.001)$.

The positive control group showed a statistically significant difference in the amount of debris remaining at all three levels of the canals in comparison to the experimental groups $(P=0.001)$. There were no significant differences among the experimental groups in this regard.

There was significantly more erosion at the coronal and middle levels than at the apical level (Mann-Whitney $U$ test; $P<0.01$ ).

EDTA caused significantly more erosion at the middle level than MTAD $(P=0.01)$. Also, EDTA resulted in more erosion at the coronal level than MTAD when subjected to ultrasonication $(P=0.04)$. Ultrasonication with EDTA significantly increased the erosive effects at the middle and coronal levels $(P=0.04, P=0.003$, respectively). Representative SEM micrographs are shown in Fig 4.

\section{Discussion}

This study was conducted to evaluate the effect of ultrasonic agitation of EDTA and MTAD on the removal of smear layer and debris, and also to determine the amount of erosion in dentinal tubules.

A recent review on the smear layer by Violich and Chandler (19) indicated that removal of the smear layer results in more thorough disinfection of the root canal system and better adaptation of filling materials to the canal walls. Various chemicals and ultrasonication have been mentioned as methods for removing this layer.

Two methods of flushing can be used during passive ultrasonic irrigation: continuous flushing of irrigant from the ultrasonic handpiece or an intermittent flush method using syringe delivery (20). A size 15 file with a fixed oscillation frequency of $30 \mathrm{kHz}$ was utilized for passive ultrasonic irrigation, as recommended by van der Sluis et al. (15).

Injecting the irrigant by syringe can control both the volume and depth of syringe penetration and the resulting flow of irrigant to the apical region of the canal system (15). On this basis, all irrigations were done using 28 -gauge

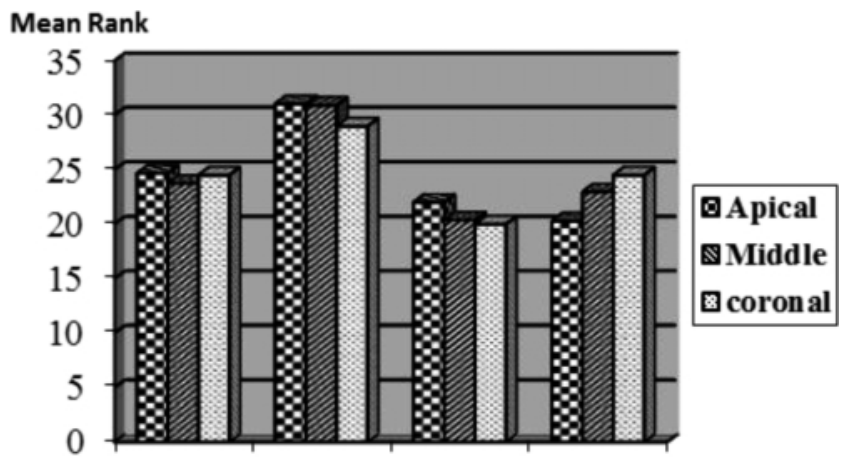

group A group B group C group D

Fig. 1 Mean rank of debris scores at the apical, middle and coronal levels in the experimental groups (Group A = EDTA, Group B = MTAD, Group C = EDTA + Ultrasonication, Group D = MTAD + Ultrasonication).

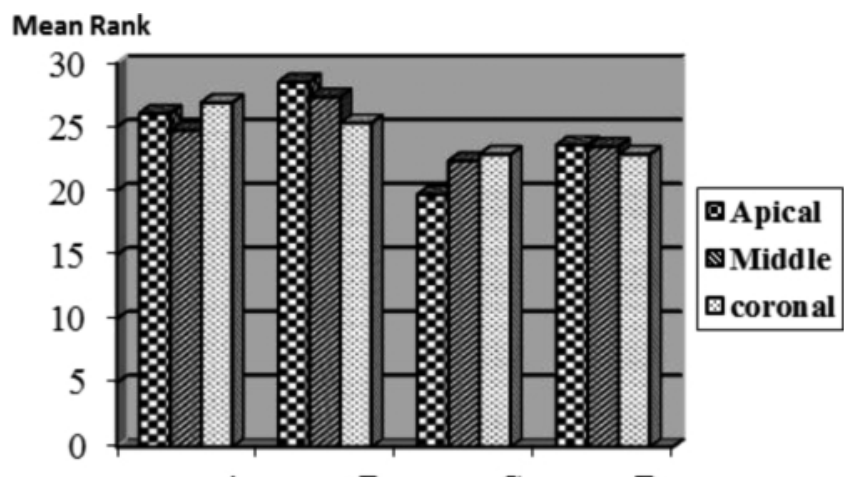

group A group B group C group D

Fig. 2 Mean rank of smear layer scores at the apical, middle and coronal levels in the experimental groups (Group $\mathrm{A}=$ EDTA, Group B = MTAD, Group C = EDTA + Ultrasonication, Group D = MTAD + Ultrasonication).

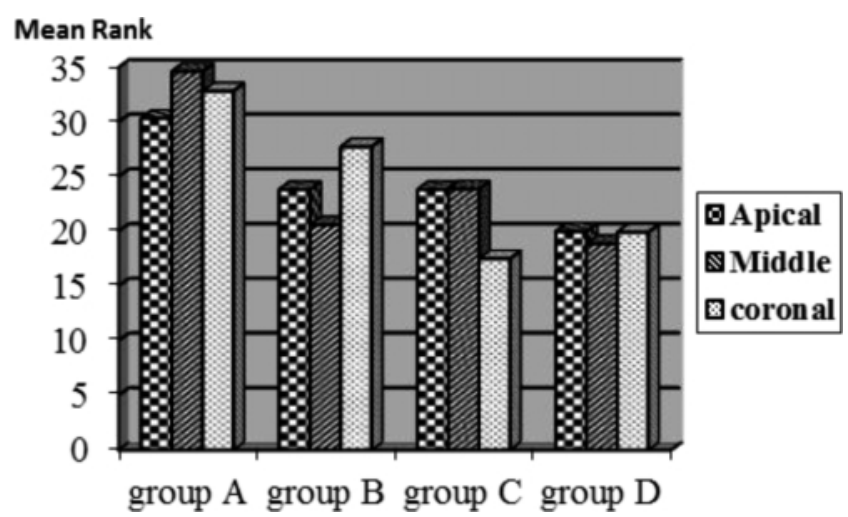

Fig. 3 Mean rank of erosion scores at the apical, middle and coronal levels in the experimental groups (Group $\mathrm{A}=$ EDTA, Group B = MTAD, Group C = EDTA + Ultrasonication, Group D = MTAD + Ultrasonication). 

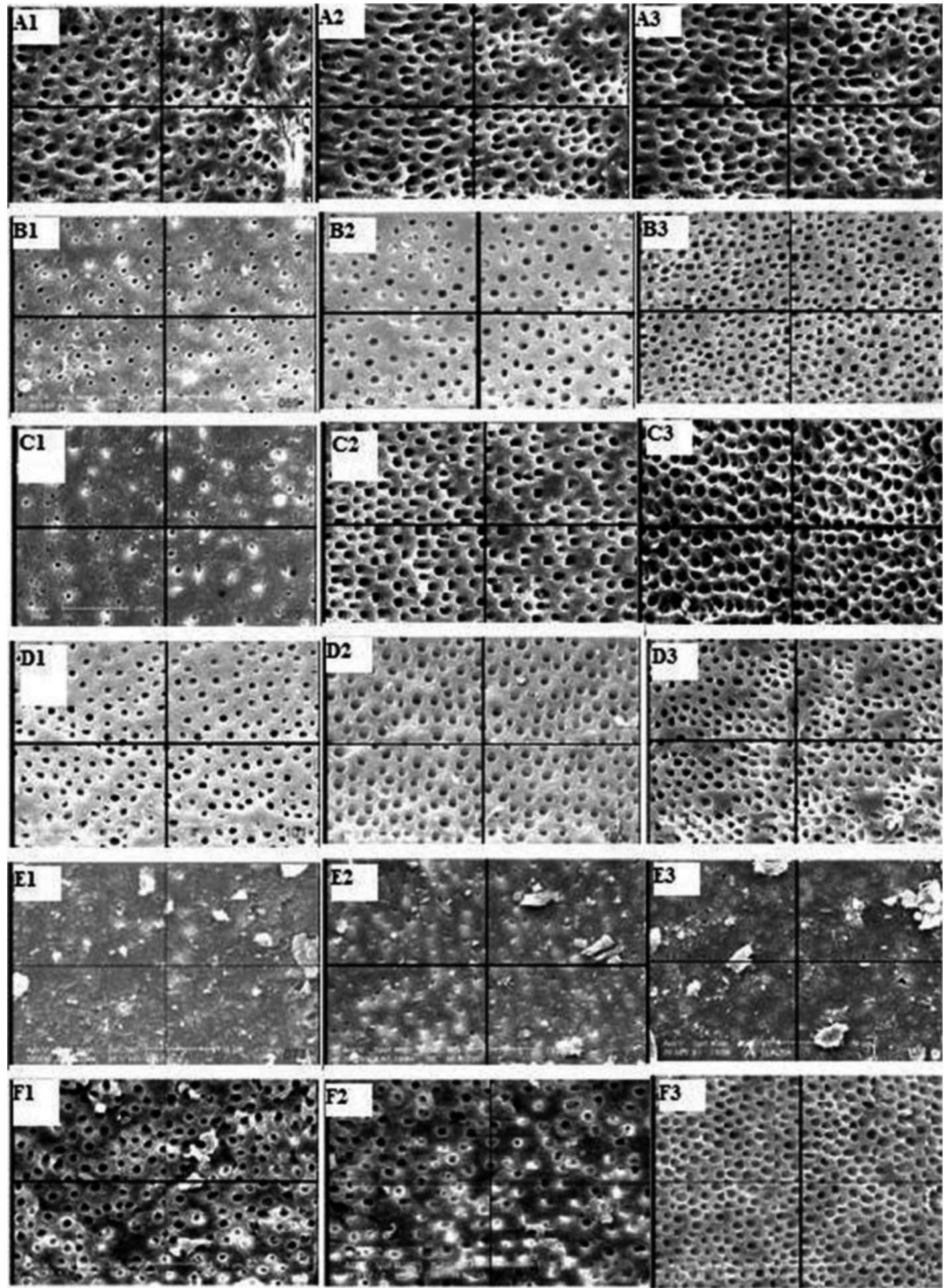

Fig. 4 SEM micrographs of the root canal wall in each group after final canal irrigation (original magnification: $\times 1,000$; scale bar is $20 \mu \mathrm{m}) . \mathrm{A}_{1}-\mathrm{F}_{1}, \mathrm{~A}_{2}-\mathrm{F}_{2}$ and $\mathrm{A}_{3}-\mathrm{F}_{3}$ show canal walls at the apical, middle and coronal levels, respectively.

In groups A and B the smear layer was removed and the peritubular and intertubular surface dentin appeared smooth.

In group $\mathrm{C}$, erosion of the peritubular and inter-tubular surface dentin was observed. Less erosion was observed in group D.

In group E (positive control group) a typical amorphous smear layer on the canal walls, and no dentinal tubule openings were observed. Clean walls were observed in group F (negative control group). 
syringes, as recommended in previous studies $(13,21)$. To mimic clinical conditions, all preparations and irrigations were done through conventional access cavities. The protocols used for each irrigant were based on previous studies (21-23).

The taper and diameter of the root canal influence the efficacy of passive ultrasonic irrigation for dentine debris removal (15). In separate studies, Lee et al. (24) and van der Sluis et al. (12) showed that increasing the canal size and taper from size 20, taper 0.04 to size 20, taper 0.08 and 0.10 resulted in more dentin debris removal by passive ultrasonic irrigation. Torabinejad et al. (1) discovered that enlarging canals up to a size 30 file allowed adequate penetration of irrigants to the apical third of the root canal. On the basis of those studies, all canals were enlarged up to size F3 Protaper, with a tip size equal to 30, taper 0.09, in the present study. We found that agitation of MTAD or EDTA resulted in better removal of the smear layer and debris, although the difference was not statistically significant. This was probably due to adequate penetration of the solution into the apical portion of the canal during irrigation. van der Sluis et al. (12) found ultrasonication to be more effective for removal of artificially placed dentin debris, although not to a significant degree. This finding was in agreement with the present results. It should also be mentioned that the sample size in this study was similar to those in previous research $(1,3,10)$.

Tay et al. (21) reported that EDTA and MTAD had equal capacities for removal of the smear layer and debris when used as the final rinse. In a recent study by Mancini et al. (25), MTAD did not completely remove the smear layer at the apical third, and the authors were not able to evaluate the degree of dentinal erosion. They used only 1 $\mathrm{ml}$ of MTAD followed by $3 \mathrm{ml}$ of $5.25 \% \mathrm{NaOCl}$, which was less than that recommended by the manufacturer and employed in our study. Also, the smaller taper of the final canal enlargement in that study (Gt\#30/.04) might have influenced the flow of irrigant into the canal.

Saghiri et al. (26) found negligible dentin erosion following application of MTAD in comparison to EDTA. In the present study, dentinal erosion was significantly higher at the middle and coronal levels within the EDTA group, in accordance with previous studies $(1,10,26)$. In a study by Torabinejad et al. (1), more abundant and larger dentinal tubules were found in the coronal third of root canals than in the middle and apical thirds. The fact that 17\% EDTA dissolves the mineral content of dentin (27) and there is free oscillation of the ultrasonic file in the coronal third, which contains large dentinal tubules, may explain the higher dentinal erosion in this region. Although some studies have used a grid to evaluate removal of the smear layer and debris $(14,28)$, the numerical evaluation scale used in the present study has been proved to be acceptable in previous work $(1,3,10,18,25)$.

Based on the findings of the present study, MTAD used in accordance with the manufacturer's protocol appears to induce less dentinal erosion with proper removal of the smear layer and debris in wide canals. Passive ultrasonic agitation of EDTA increases dentin erosion and does not seem to be needed in large canals.

\section{References}

1. Torabinejad M, Khademi AA, Babagoli J, Cho Y, Johnson WB, Bozhilov K, Kim J, Shabahang S (2003) A new solution for the removal of the smear layer. J Endod 29, 170-175.

2. Hata G, Hayami S, Weine FS, Toda T (2001) Effectiveness of oxidative potential water as a root canal irrigant. Int Endod J 34, 308-317.

3. Lui JN, Kuah HG, Chen NN (2007) Effect of EDTA with and without surfactants or ultrasonics on removal of smear layer. J Endod 33, 472-475.

4. Yamada RS, Armas A, Goldman M, Lin PS (1983) A scanning electron microscopic comparison of a high volume final flush with several irrigating solutions: Part 3. J Endod 9, 137-142.

5. Calt S, Serper A (2002) Time-dependent effects of EDTA on dentin structures. J Endod 28, 17-19.

6. Niu W, Yoshioka T, Kobayashi C, Suda H (2002) A scanning electron microscopic study of dentinal erosion by final irrigation with EDTA and $\mathrm{NaOCl}$ solutions. Int Endod J 35, 934-939.

7. Sen BH, Ertürk O, Pişkin B (2009) The effect of different concentrations of EDTA on instrumented root canal walls. Oral Surg Oral Med Oral Pathol Oral Radiol Endod 108, 622-627.

8. Newberry BM, Shabahang S, Johnson N, Aprecio RM, Torabinejad M (2007) The antimicrobial effect of biopure MTAD on eight strains of Enterococcus faecalis: an in vitro investigation. J Endod 33, 13521354.

9. Shabahang S, Torabinejad M (2003) Effect of MTAD on Enterococcus faecalis-contaminated root canals of extracted human teeth. J Endod 29, 576-579.

10. Torabinejad M, Cho Y, Khademi AA, Bakland LK, Shabahang S (2003) The effect of various concentrations of sodium hypochlorite on the ability of MTAD to remove the smear layer. J Endod 29, 233-239.

11. Baker NA, Eleazer PD, Averbach RE, Seltzer S (1975) Scanning electron microscopic study of the efficacy of various irrigating solutions. J Endod 1, 
127-135.

12. van der Sluis LW, Wu MK, Wesselink PR (2005)The efficacy of ultrasonic irrigation to remove artificially placed dentine debris from human root canals prepared using instruments of varying taper. Int Endod J 38, 764-768.

13. Plotino G, Pameijer CH, Grande NM, Somma F (2007) Ultrasonics in endodontics: a review of the literature. J Endod 33, 81-95.

14. Passarinho-Neto JG, Marchesan MA, Ferreira RB, Silva RG, Silva-Sousa YT, Sousa-Neto MD (2006) In vitro evaluation of endodontic debris removal as obtained by rotary instrumentation coupled with ultrasonic irrigation. Aust Endod J 32,123-128.

15. van der Sluis LW, Versluis M, Wu MK, Wesselink PR (2007) Passive ultrasonic irrigation of the root canal: a review of the literature. Int Endod J 40, 415426.

16. Gutarts R, Nusstein J, Reader A, Beck M (2005) In vivo debridement efficacy of ultrasonic irrigation following hand-rotary instrumentation in human mandibular molars. J Endod 31, 166-170.

17. Schneider SW (1971) A comparison of canal preparations in straight and curved root canals. Oral Surg Oral Med Oral Pathol 32, 271-275.

18. Schäfer E, Lohmann D (2002) Efficiency of rotary nickel-titanium FlexMaster instruments compared with stainless steel hand K-Flexofile - Part 2. Cleaning effectiveness and instrumentation results in severely curved root canals of extracted teeth. Int Endod J 35, 514-521.

19. Violich DR, Chandler NP (2010) The smear layer in endodontics - a review. Int Endod J 43, 2-15.

20. Cameron JA (1988) The effect of ultrasonic endodontics on the temperature of the root canal wall. J Endod 14, 554-559.
21. Tay FR, Pashley DH, Loushine RJ, Doyle MD, Gillespie WT, Weller RN, King NM (2006) Ultrastructure of smear layer-covered intraradicular dentin after irrigation with BioPure MTAD. J Endod $32,218-221$.

22. Park DS, Torabinejad M, Shabahang S (2004) The effect of MTAD on the coronal leakage of obturated root canals. J Endod 30, 890-892.

23. Crumpton BJ, Goodell GG, McClanahan SB (2005) Effects on smear layer and debris removal with varying volumes of $17 \%$ REDTA after rotary instrumentation. J Endod 31, 536-538.

24. Lee SJ, Wu MK, Wesselink PR (2004) The efficacy of ultrasonic irrigation to remove artificially placed dentine debris from different-sized simulated plastic root canals. Int Endod J 37, 607-612.

25. Mancini M, Armellin E, Casaglia A, Cerroni L, Cianconi L (2009) A comparative study of smear layer removal and erosion in apical intraradicular dentine with three irrigating solutions: a scanning electron microscopy evaluation. J Endod 35, 900903.

26. Saghiri MA, Delvarani A, Mehrvarzfar P, Malganji G, Lotfi M, Dadresanfar B, Saghiri AM, Dadvand $S$ (2009) A study of the relation between erosion and microhardness of root canal dentin. Oral Surg Oral Med Oral Pathol Oral Radiol Endod 108, e29-34.

27. Beltz RE, Torabinejad M, Pouresmail M (2003) Quantitative analysis of the solubilizing action of MTAD, sodium hypochlorite, and EDTA on bovine pulp and dentin. J Endod 29, 334-337.

28. Mayer BE, Peters OA, Barbakow F (2002) Effects of rotary instruments and ultrasonic irrigation on debris and smear layer scores: a scaning electron microscopic study. Int Endod J 35, 582-589. 